\title{
Variáveis dos gastos públicos de uma universidade sobre o crescimento econômico dos seus municípios de inserção
}

\section{Variables of a university's public spending on the economic growth of its insertion municipalities}

\author{
ESDRAS ANTUNES DO NASCIMENTOD
}

RODRIGO GARCIA DUARTE (D)

\section{RESUMO}

Este trabalho analisou o comportamento dos gastos públicos educacionais executados pela Universidade Estadual do Sudoeste da Bahia (UESB), buscando evidências significativas das relações desses gastos com o Produto Interno Bruto - PIB e com os Valores Adicionados - VA's dos setores econômicos dos municípios baianos de Vitória da Conquista, Jequié e Itapetinga. Teoricamente se discute que as universidades exercem influência sobre o desenvolvimento econômico regional, em razão das vantagens provenientes das externalidades produzidas (spillovers), passíveis de verificação por meio dos efeitos multiplicadores dos gastos com pessoal, custeio e investimentos sobre os municípios, que provêm a comunidade regional com conhecimento, pesquisa e inovação. Além disso, afirma-se que as universidades impactam os setores produtivos das suas regiões de inserção, dada a sua força motriz na atração de novos negócios e ao fomento endógeno de novos empreendimentos ligados à indústria, comércio e serviços decorrentes das suas atividades e estímulos. Trata-se de um estudo quantitativo, de caráter descritivo e exploratório, instrumentalizado por meio de dados secundários, disponibilizados em bancos de dados de instituições públicas, fundamentado nas teorias dos gastos públicos e do desenvolvimento regional. $\mathrm{O}$ resultado, a partir das análises estatísticas, indicou que apesar da 
relevância dos gastos públicos realizados pela UESB em relação aos seus municípios de inserção, as taxas de crescimento destes gastos não possuem correlação siginificativa e direta com as taxas de crescimento do PIB e com as taxas de crescimento dos VA's dos setores econômicos dos municípios baianos de Vitória da Conquista, Jequié e Itapetinga.

Palavras-chave: Gastos Públicos, Universidade e Desenvolvimento Regional.

\section{Abstract}

This work analyzed the behavior of public educational expenditures carried out by the State University of Southwest Bahia (UESB), looking for significant evidence of the relationship of these expenditures with the Gross Domestic Product - GDP and with the Added Values - VA's of the economic sectors of the municipalities of Vitória in Bahia da Conquista, Jequié and Itapetinga. Theoretically, it is argued that universities exert an influence on regional economic development, due to the advantages arising from the externalities produced (spillovers), which can be verified through the multiplier effects of personnel expenses, funding and investments on the municipalities, which come from the community. with knowledge, research and innovation. In addition, it is said that universities impact the productive sectors of their insertion regions, given their driving force in attracting new businesses and the endogenous encouragement of new ventures related to industry, commerce and services resulting from their activities and incentives. This is a quantitative, descriptive and exploratory study, using secondary data, made available in databases of public institutions, based on theories of public spending and regional development. The result, based on statistical analysis, indicated that despite the relevance of public expenditures made by UESB in relation to its insertion municipalities, the growth rates of these expenditures have no significant and direct correlation with the GDP growth rates and with the growth rates of VA's in the economic sectors of the Bahia municipalities of Vitória da Conquista, Jequié and Itapetinga.

Keywords: Public Spending, University and Regional Development. 


\section{INTRODUÇÃo}

Nas últimas décadas, a sociedade tem exigido cada vez mais transparência, eficiência, eficácia e efetividade das organizações públicas, espeficicamente das universidades e dos munícipios (RAMÍREZ-GUTIÉRREZ et al, 2020). Uma das possibilidades de verificação dessas prerrogativas pode se dar através da análise dos gastos públicos e seus efeitos no desenvolvimento regional, estratégia utilizada para a elaboração deste estudo.

As universidades públicas são importantes instrumentos de promoção e consolidação das políticas educacionais em nível nacional e regional, pelas contribuições que desenvolvem para com o ensino superior (graduação e pós-graduação), além da pesquisa e extensão, bem como pela articulação com o processo de formação continuada da educação básica (MOTA; GONÇALVES, 2020). Atreladas à função alocativa de governo, as universidades atuam como interlocutoras dessas políticas públicas de educação superior em face da comunidade regional, realizando a aplicação de recursos públicos como mecanismo para geração de emprego e renda.

Nesse sentido, este estudo parte da premissa que as organizações universitárias, além de proporcionarem a formação e qualificação de recursos humanos, também contribuem para o desenvolvimento econômico dos municípios, sobretudo, daqueles que compõem suas áreas de abrangência territorial, precisamente, na distribuição das finanças públicas. Para efeito de verificação empírica, selecionamos para análise o caso da Universidade Estadual do Sudoeste da Bahia (UESB), uma instituição multicampi, com localização em três municípios - Vitória da Conquista, Jequié e Itapetinga, atuando há quatro décadas no sudoeste baiano.

Isso posto, face a premissa apresentada, considerando a literatura existente, questionamos: Qual a relação entre o comportamento do gasto público realizado pela UESB e os indicadores econômicos dos municípios baianos de Vitória da Conquista, Jequié e Itapetinga (Produto Interno Bruto - PIB e Valores Adicionados - VA's dos setores econômicos)? Considerando o problema evidenciado, busca-se neste trabalho analisar a relação entre o comportamento dos gastos públicos da UESB e os indicadores econômicos dos municípios baianos de Vitória da Conquista, Jequié e Itapetinga 
(Produto Interno Bruto - PIB e Valores Adicionados - VA's dos setores econômicos).

O trabalho, além desta introdução, está estruturado em quatro seções: a primeira traz uma síntese das discussões teóricas sobre gastos públicos sociais e seus efeitos no desenvolvimento regional, com ênfase em gastos com educação superior; a segunda versa sobre as estratégias metodológicas escolhidas para o desenvolvimento da investigação; a terceira evidencia os resultados do estudo, destacando a análise da correlação entre os gastos públicos da UESB, PIB e VA's dos municípios e, por fim, as considerações finais sobre o estudo que trazem ao debate o papel da universidade como importante agente do sistema econômico local, apesar dos resultados estatísticos evidenciados.

\section{REFERENCIAL TEÓRICO}

\subsection{Efeitos dos Gastos Públicos no Desenvolvimento Regional: Estado da Arte}

Este tópico apresenta uma síntese das discussões teóricas sobre gastos públicos e seus efeitos no desenvolvimento regional, sob a ótica das implicações econômicas geradas por uma universidade pública. Para tanto, com o intuito de evidenciar o estado da arte sobre o campo de estudo, foram realizadas consultas às bases de dados dos periódicos Scientific Periodicals Electronic Library (Spell) e SciVerse Scopus (Scopus), em relação aos últimos seis anos.

Preliminarmente, foram evidenciadas as principais correntes teóricas sobre o desenvolvimento. Conforme revisão bibliográfica realizada por Bellingieri (2017), o debate sobre o desenvolvimento se sustenta nas escolas das Teorias Clássicas da Localização e das Teorias do Desenvolvimento Regional (Base de Exportação, Causação Circular Cumulativa, Desenvolvimento Desigual e Transmissão Inter-regional do Crescimento, Polos de Crescimento), bem como nas Teorias de Desenvolvimento Local, vinculadas ao paradigma do desenvolvimento endógeno (Nova Teoria do Crescimento, Distrito Industrial, Milieu Innovateur, Cluster, Capital Social, Cidade Criativa).

Em face dessa retomada teórica, Bellingieri (2017, p. 7) aponta para uma vasta literatura que percorre por diversos campos de es- 
tudo (Economia, Sociologia, Geografia e etc.) na investigação sobre fenômeno do desenvolvimento (o que é, como e por que ocorre, e como, e se, pode ser medido). Objeto de análise disciplinar no campo da administração, o desenvolvimento tem sido concebido como saber disperso e fragmentado que sobrevive em diversos campos do conhecimento científico (SANTOS; SANTOS; BRAGA, 2016, p. 281).

Santos (2014, p. 74), ao abordar sobre a influência do termo no pensamento moderno em face ao comportamento humano, explana que o conceito de desenvolvimento é fruto de uma construção histórica sustentada em três visões paradigmáticas, as quais tornam possível a compreensão do fenômeno: desenvolvimento como crescimento econômico, desenvolvimento como satisfação das necessidades básicas e desenvolvimento como elemento de sustentabilidade socioambiental.

No que concerne as suas raízes, o debate sobre o desenvolvimento tem seu início na economia, a partir das contribuições de Adam Smith (1776), Thomaz Malthus (1798), David Ricardo (1817) e Karl Marx (1867) com fundamento no acúmulo de riquezas perante o estrato de uma sociedade industrial (SANTOS, 2014), e, historicamente, tem incorporado em seu conceito aspectos relativos as novas configurações políticas e intelectuais, coexistindo em um debate multiparadigmático (BELLINGIERI, 2017).

Ante a influência da escola keynesiana, que enfatiza os fatores aglomerativos, as bases para o desenvolvimento têm sido estabelecidas sob a perspectiva de uma melhor utilização do acúmulo de riquezas (crescimento) para a promoção do bem estar social, junto ao conjunto da população de um país ou região, confirmando uma dimensão social para o fenômeno (BELLINGIERI, 2017). Nesse sentido, ressalta-se que o crescimento econômico, apesar de necessário, não é suficiente para que ocorra o desenvolvimento (BRESSER-PEREIRA, 2019).

No campo da causalidade, Bellingieri (2017) aborda que o desenvolvimento pode se relacionar com fatores externos ou exógenos, tais como a instalação de grandes empresas ou projetos governamentais, bem como determinar ou sofrer a influência de outros fenômenos. Amparado por Santos (2006, p. 106-7), Bellingieri (2017) salienta que "nenhum acontecimento pode ser sozinho, 
nem completamente, a causa de outro evento" quando se trata de desenvolvimento.

Esse pressuposto reforça que a mensuração do desenvolvimento deve considerar a análise de aspectos quantitativos, comparando números com indicadores do Produto Interno Bruto - PIB (crescimento), por exemplo, em associação com aspectos qualitativos, com relevância para as questões sociais (desenvolvimento). Nesse interim, a causalidade para o desenvolvimento, em que pese o seu caráter social, pode ser observado sob a lente da educação superior (universidades), importante instrumento que influencia melhoria da qualidade de vida, a partir da formação/qualificação de pessoas e da disseminação do conhecimento (MOTA; GONÇALVES, 2020).

As abordagens teóricas sobre a teoria dos pólos de crescimento, por sua vez, contribuem para o desenvolvimento regional a partir da explicação de como se comportam os agentes em mercados não competitivos (JESUS; SPINOLA, 2015). Com base nos pressupostos de Perroux, Boudeville e Paelinck, essa abordagem parte da ideia de que o crescimento econômico se realiza de maneira desequilibrada, em decorrência do encadeamento dos efeitos de forças motrizes em torno de um espaço econômico e geográfico (JESUS; SPINOLA, 2015).

Deste modo, argumenta-se que um polo é formado a partir do surgimento de uma unidade econômica, ou seja, manifesta-se em pontos ou polos específicos e, sucessivamente, se amplia, para toda a economia em vista das vantagens da aglomeração (JESUS; SPINOLA, 2015). Tal premissa auxilia na compreensão das realidades regionais que evidenciam ritmos de crescimento diferenciado umas das outras, onde as cidades e/ou regiões de menor porte dependem da estrutura de certos polos e dos recursos distribuídos em certas regiões.

Evidências no campo teórico também apresentam uma discussão clássica marshalliana (Marshall, Arrow, Romer e Jacobs) sobre os efeitos da atuação de agentes externos, referenciado como externalidades (BEZERRA et al., 2018). No campo da teoria econômica regional, as externalidades indicam os impactos positivos (spillovers) e negativos (efeitos adversos) sobrepostos de uma entidade (pessoa física e/ou jurídica) para outra, tal como uma universidade em relação ao seu município de inserção, em determinado espaço 
produtivo, de maneira não intencional, em detrimento do consumo ou produção realizados, (BEZERRA et al., 2018).

Esse debate parte da notabilização do desenvolvimento econômico desequilibrado, em razão das falhas de mercado e dos equívocos na adoção de certas políticas públicas para a redução de desigualdades peculiares de cada região. Nesse sentido, debate-se que uma das formas de dirimir as desigualdades sociais se sustenta no controle sobre os efeitos negativos das externalidades, provocadas pelo mercado ou pelos próprios agentes públicos, por meio de políticas públicas que repercutam em alocação de recursos mais eficientes (BEZERRA et al., 2018).

Contribuindo sobre outro prisma, a teoria do desenvolvimento endógeno surge para incitar o desenvolvimento por meio do fortalecimento da ambiência interna, fomentando valorização do território e dos atores locais e ao desenvolvimento de baixo para cima, em contraposição ao de cima para baixo (BELLINGIERI, 2017). Essa abordagem contribui para o desenvolvimento regional, sob a ótica contemporânea da territorialidade, governança e pertencimento, frente ao debate político sobre as potencialidades produtivas e competitivas de cidades e/ou regiões, em um processo onde o conhecimento e a inovação se tornam elementos essenciais (ALMEIDA, 2020).

Portanto, as universidades, e seu papel no desenvolvimento, podem ser pensadas na perspectiva da sua inclusão nos sistemas regionais, não apenas como um agente externo, mas como um ator a ser observado (PANIZZI; MEIRELLES; HOPPE, 2018). Nesse campo, depreende-se que estudar e pensar as cidades e o seu desenvolvimento, perpassa por uma compreensão de sistemas tidos dinâmicos, complexos e adaptativos que sofrem influências da transferência de conhecimento e da inovação provenientes das universidades (PANIZZI; MEIRELLES; HOPPE, 2018; ALMEIDA, 2020).

No cerne das abordagens teóricas, as universidades podem ser concebidas como unidades econômicas que, por meio das suas atividades de ensino, pesquisa e extensão comunitária, geram atratividade para cidades e regiões próximas, favorecendo o encadeando atividades subjacentes via processo de aglomeração (BONHO, 2020). Além disso, pela disponibilização de suporte científico e tecnológico, 
além do desempenho de uma terceira missão voltada para a inovação, as universidades contribuem para a atração de novos negócios, bem como para o surgimento endógeno de novos empreendimentos (ALMEIDA, 2020).

As evidências das implicações socioeconômicas das universidades nos territórios, por sua vez, podem ser avaliadas a partir do gasto público realizado (ALLMEIDA, 2020). O gasto público é tratado nas finanças públicas como provenientes da execução das despesas orçamentárias públicas, sendo analisado sob a ótica dos gastos correntes (pessoal, encargos e custeio), bem como dos gastos de capital (investimentos), ou ainda, interpretado em detrimento de suas funções e subfunções (MTO, 2020).

Segundo Fittipaldi, Costa e Araújo (2017), o gasto público, classificado por função de Estado, pode ser concebido pelo: a) gasto mínimo, que se relaciona com os dispêndios básicos de governo; b) gasto econômico, que visa a adoção de medidas frente as interfaces de mercado; e c) gasto público social, atrelado aos investimentos realizados nas áreas de educação, saúde, moradia, cultura, saneamento, assistência social e previdência social, conforme também indica Reis e Bueno (2019).

Em relação aos efeitos dos gastos públicos, Reis e Bueno (2019), a partir da investigação dos efeitos dos gastos públicos sociais no crescimento econômico, destacam duas tendências principais em debate na literatura: a) uma frente que defende a aplicação direta de recursos no combate a vulnerabilidade de populações pobres; e b) outra vista que sustenta a educação enquanto investimento que incrementa a produtividade das economias e, consequentemente, a qualidade de vida das pessoas.

Segundo Brandão (2019), pesquisas recentes no campo do desenvolvimento regional brasileiro, como as investigações realizadas por Resende (2014) e Monteiro Neto (2015), revelam que as diminuições das desigualdades regionais também se pautam em políticas não explicitamente regionais, como as sociais, o que reforça a importância da alocação de recursos com o gasto público social, como na educação superior.

Tendo em vista o efeitos dos gastos sobre os indicadores socioeconômicos, Costa e Gartner (2017), destacam a importância da 
alocação de recursos de governo, a partir da provisão de bens semipúblicos, como a educação, ao citarem vários estudos que relacionaram os gastos públicos com o impacto sobre a redução das desigualdades de renda e emprego. Os gastos públicos sociais, relacionados com a educação e cultura estaduais, também são notabilizados por Hiromoto (2018), em relação às variáveis que apresentam melhores resultados com a diminuição dos índices de pobreza.

As universidades geram impactos econômicos de curto prazo, que podem ser analisados a partir da movimentação de recursos financeiros (ALMEIDA, 2020). Os impactos diretos da atuação das universidades, relacionados as externalidade positivas (spillovers), podem ser observadas sobre algumas formas: a) gastos com pessoal das universidades, provenientes do pagamento de salários a servidores e colaboradores; e b) gastos para a manutenção e expansão das universidades, que se traduzem nas despesas com custeio e investimentos em obras e equipamentos.

Outra perspectiva que pode ser evidenciada são os efeitos do gasto público das universidades em relação às transformações econômicas provenientes do crescimento do Produto Interno Bruto - PIB dos municípios de inserção, bem como por meio da sua participação no valor adicionado das atividades econômicas. Essa perspectiva avalia a participação da universidade no sistema econômico local (ROMANATTO et al., 2015).

Portanto, a presente pesquisa, ao admitir o papel das universidades no desenvolvimento regional, seja na perspectiva dos polos de crescimento, das externalidades e dos fatores endógenos, considera ser possível evidenciar empiricamente alguns resultados dessas contribuições avaliando o gasto público social realizado pela UESB em comparação aos números dos municípios onde esta se articula, conforme análises expostas na seção de resultados.

Para tanto, o gasto público da UESB foi definido como variável dependente do estudo (ROMANATTO et al., 2015; ALMEIDA, 2020) e os indicadores do PIB e VA's dos municípios foram delimitados como variáveis independentes (DALEY; GARAND, 2005; BUENO, 2013; GUANDALINI, 2016). 


\section{Metodologia}

Em adequação ao objetivo proposto, esta pesquisa possui uma abordagem quantitativa, com caráter descritivo e exploratório (GIL, 2009). É descritiva dado ao interesse de descrever a variável que envolve o gasto público realizado por uma universidade estadual. É exploratória porque analisa a correlação destes gastos com o PIB e os valores adicionados dos setores econômicos dos seus municípios de inserção, a saber Vitória da Conquista (BA), Jequié (BA) e Itapetinga (BA).

O estudo de caso foi definido como estratégia de condução da pesquisa visando um maior aprofundamento sobre a realidade de uma universidade pública no contexto econômico dos municípios onde ela atua diretamente, considerando a dinâmica de crescimento econômico em geral e do desempenho dos setores produtivos em particular (YIN, 2015). O caso objeto de estudo é a Universidade Estadual do Sudoeste da Bahia - UESB, escolhida em razão da sua trajetória e relevância no sudoeste baiano, um vez que trata-se da primeira instituição de ensino superior, pública na região, com quatro décadas de existência.

A coleta de dados secundários ocorreu por meio de relatório de execução orçamentária, valores pagos pela UESB, além dos valores dos PIBs dos municípios baianos, deflacionados com base no deflator oficial do IBGE (2020). Foi observado um recorte temporal para análise dos dados equivalente ao período de 2013 a 2018, considerando a migração dos dados orçamentários da UESB para o sistema FIPLAN ao final de 2012, por medida de adequação da contabilidade do estado da Bahia às novas normas de contabilidade internacional aplicadas ao setor público, bem como levando em conta os dados dos PIBs municipais disponíveis até o ano de 2018.

Cumpre salientar que, como medida do crescimento econômico, utilizou-se o Produto Interno Bruto, bem como os valores adicionados dos setores econômicos individualmente, sendo esta a variável mais comum para mensuração da atividade econômica agregada, representando o total de bens e serviços finais produzidos dentro de um país, estado ou cidade em determinado período (ABEL; BERNANKE; CROUSHORE, 2008). As variáveis, os tipos de dados, as fontes e procedimentos de coleta e as finalidades de uso são 
demonstrados nas medidas operacionais utilizadas representadas no Quadro 1.

Quadro 1 - Medidas operacionais utilizadas na pesquisa

\begin{tabular}{|c|c|c|}
\hline Tipos de dados & $\begin{array}{l}\text { Fontes e procedimetnos de } \\
\text { coleta }\end{array}$ & Finalidade \\
\hline $\begin{array}{l}\text { Gastos públicos rea- } \\
\text { lizados pela UESB }\end{array}$ & $\begin{array}{l}\text { Extração dos dados por } \\
\text { meio do Portal da Trans- } \\
\text { parência do Governo do } \\
\text { Estado da Bahia e Sistema } \\
\text { Corporativo Integrado de } \\
\text { Planejamento, Contabilida- } \\
\text { de e Finanças do Estado da } \\
\text { Bahia (FIPLAN). }\end{array}$ & $\begin{array}{l}\text { Especificar a taxa de } \\
\text { crescimento dos gas- } \\
\text { tos públicos despendi- } \\
\text { dos a cada ano. }\end{array}$ \\
\hline Deflator oficial IPCA & $\begin{array}{l}\text { Cálculo realizado a partir } \\
\text { do IBGE }(2020)\end{array}$ & $\begin{array}{l}\text { Deflacionar as séries } \\
\text { de dados aos valores } \\
\text { de preços do ano/base } \\
\text { dez/2018. }\end{array}$ \\
\hline $\begin{array}{l}\text { PIB total dos muni- } \\
\text { cípios baianos de Vi- } \\
\text { tória da Conquista, } \\
\text { Jequié e Itapetinga. }\end{array}$ & $\begin{array}{l}\text { Aferição do PIB nominal } \\
\text { total, por município e ano, } \\
\text { a partir dos dados da Su- } \\
\text { perintendência de Estudos } \\
\text { Econômicos e Sociais do } \\
\text { Estado da Bahia (SEPLAN, } \\
\text { 2019) e IBGE (2020). }\end{array}$ & $\begin{array}{l}\text { Apurar a taxa de cres- } \\
\text { cimento econômico do } \\
\text { PIB a cada ano. }\end{array}$ \\
\hline $\begin{array}{l}\text { Valor adicionado } \\
\text { bruto dos setores } \\
\text { agropecuária, indús- } \\
\text { tria, serviços e admi- } \\
\text { nistração público por } \\
\text { município. }\end{array}$ & $\begin{array}{l}\text { Aferição do PIB total e } \\
\text { valor adicionado corrente } \\
\text { total, por município e ano, } \\
\text { a partir dos dados da Su- } \\
\text { perintendência de Estudos } \\
\text { Econômicos e Sociais do } \\
\text { Estado da Bahia (SEPLAN, } \\
\text { 2019) e IBGE (2020). }\end{array}$ & $\begin{array}{l}\text { Apurar a taxa de cres- } \\
\text { cimento econômico } \\
\text { dos setores produtivos } \\
\text { a cada ano. }\end{array}$ \\
\hline $\begin{array}{l}\text { Deflator oficial do } \\
\text { PIB }\end{array}$ & $\begin{array}{l}\text { Cálculo realizado a partir } \\
\text { do IBGE }(2020)\end{array}$ & $\begin{array}{l}\text { Deflacionar as séries } \\
\text { de dados dos valores } \\
\text { nominais à valores } \\
\text { reais em cada ano. }\end{array}$ \\
\hline
\end{tabular}

Fonte: Elaborado pelos autores. 


\subsection{Métodos e testes empregados na pesquisa}

Nessa seção são detalhados os procedimentos para tratamento e análise dos dados empregados na pesquisa. Destaca-se que as análises foram realizadas por meio do software SPSS® versão 23.0.

\subsubsection{Verificação da Normalidade de Distribuição dos Dados}

A avaliação da normalidade de distribuição dos dados é primordial para a adequada descrição da amostra e sua análise inferencial, sustentando os modelos teóricos nos principais testes estatísticos empregados na análise de dados (NORMAN; STREINER, 2014).

De acordo Lopes, Branco e Soares (2013), existe uma gama de métodos estatísticos para medir a distribuição normal, a partir das inferências estatísticas de estimação e testes de hipóteses, tais como Anderson-Darling, Cramer-Von Mises, D'Agosti-no-Pearson, Jarque-Bera, Kolmogorov-Smirnov e Shapiro-Wilk, além de recursos gráficos, como histograma e normal Quantil-Quantil (Q-Q Plot), bem como os dados de assimetria e curtose.

Os testes de Kolmogorov-Smirnov e Shapiro-Wilk são muito utilizados em pesquisas científicas como critérios estatísticos para definição do método de correlação mais apropriado para os dados em análise (LEOTTI; BIRCK; RIBOLDI, 2005). Ambos os testes tendem a fornecer resultados homogêneos entre si a partir do teste de hipótese estatísticas dadas por: H0 (dados que seguem uma distribuição normal de Gauss); e H1 (dados que não seguem uma distribuição normal de Gauss).

Os testes de Kolmogorov-Smirnov e Shapiro-Wilk, fornecem o parâmetro valor de prova (valor-p, p-value ou significância). Assim, ao processar esses testes, espera-se que o nível de significância indique um $\mathrm{p}>0,05$, interpretando-o como medida do grau de concordância entre os dados e a hipótese nula (H0), sendo acatada a distribuição de normalidade dos dados (CIRILLO; FERREIRA, 2003; ÖZTUNA, ELHAN; TÜCCAR 2006).

Graficamente, a distribuição da normalidade pode ser verificada por meio do gráfico Quantil-Quantil ou Q-Q Plot, considerada a melhor forma de se verificar a linearidade da relação entre duas variáveis (ESPIRITO-SANTO; DANIEL, 2017). O gráfico permite uma visualização do eixo horizontal com os valores observados das 
variáveis, bem como do eixo vertical com os valores esperados em caso de distribuição normal. Se há uma boa aderência dos dados à distribuição normal os pontos estão próximos a reta de referência apresentada no gráfico (LEOTTI; COSTER; RIBOLDI, 2012).

Outro método empregado para identificar as características da distribuição e verificar a normalidade dos dados deriva da análise do escore $Z$ (score Z) calculado sobre a divisão dos valores estatísticos da assimetria (skewness) e da curtose (kurtosis) pelo erro padrão, separadamente (TABACHNICK; FIDEll, 2007; PALLANT, 2011). A normalidade dos dados é confirmada quando os valores do escore $\mathrm{Z}$ atendem aos seguintes critérios:

- Valor maior que $|1.96|$ é significativo $p<0,05$;

- Valor acima que $|2.58|$ é significativo $p<0,01$;

- Valor acima que $|3.29|$ é significativo $p<0,001$.

Os testes de Kolmogorov-Smirnov e Shapiro-Wilk, o gráfico Q-Q Plot e o escore Z (score Z) provenientes dos valores da assimetria (skewness) e da curtose (kurtosis), forma elegidos para verificação da normalidade dos dados na presente pesquisa.

\subsubsection{Deflacionamento dos Gastos da UESB e do PIB}

O deflacionamento dos valores orçamentários executados pela UESB foram realizados em razão do Índice de Preços ao Consumidor Amplo (IPCA), ano base 2018 (IBGE, 2020), para fins de análise dos valores correntes (FEITOSA; HEIN, 2014).

O deflacionamento das séries de dados do PIB e dos valores adicionados dos setores econômicos dos municípios baianos analisados foram realizados conforme metodologia do IBGE (2020), considerando a taxa de crescimento do PIB apurada em razão do ano base 2018 .

A metodologia do IBGE calcula a taxa de crescimento do PIB com base no PIB real do ano base e do ano anterior, conforme fórmula a seguir: 
Taxa de Crescimento ano atual $=$ PIB Real ${ }_{\text {ano atual }}-$ PIB Real $_{\text {ano anterior }}$

PIB Real ano anterior

Para tanto, com base na indicação da taxa de crescimento do ano base, apura-se o valor do PIB real do ano anterior, como segue:

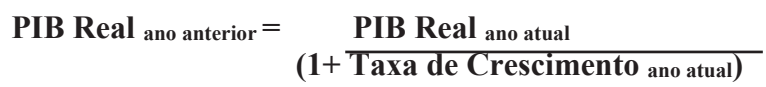

Por fim, o cálculo do deflator é apurado considerando o PIB nominal e o PIB real do ano em análise, através da fórmula apresentada abaixo:

$$
\text { Deflator }=\frac{(\text { PIB Nominal } \text { ano analisado }) \times 100}{\text { PIB Real ano analisado }}
$$

\subsubsection{Coeficientes de Correlação}

São vários os coeficientes de correlação empregados em pesquisas, cujos usos dependem da escala de medida utilizada, da forma da relação e, até mesmo, em razão do volume amostral, uma vez que o valor de $p$ é afetado pelo tamanho da amostra (ESPIRITO-SANTO; DANIEL, 2017). Esses coeficientes são técnicas estatísticas que mensuram a força de associação entre as variáveis e o que elas representam (FIELD, 2009).

Os principais coeficientes de correlação utilizados em estudos são o Coeficiente de Pearson e o Coeficiente de Spearman. O Coeficiente de correlação de Spearman, escolhido para análise nesta pesquisa, apresenta-se como uma medida para variáveis ordinais equivalente ao coeficiente de correlação de Pearson, aplicado a dados ordenados (GUIMARÃES, 2017). A equação matemática para análise da correlação por postos de Spearman é dada pela seguinte fórmula:

$$
r_{s}=1-\frac{6 \sum_{i=1}^{n} d_{i}^{2}}{N^{3}-N}
$$

As medidas do ró de Spearman variam entre -1 e 1, igualmente à correlação de Pearson, e consiste na correlação entre os postos de dois conjuntos de observações, ou seja, uma verificação a partir da posição ordinal da observação ou do caso na variável (COHEN; COHEN, 1983). Os parâmetros para análise do coeficiente de Spearman são definidos por Finney (1980): 
- Resultados entre 10,90 e $1 \mid$ caracterizam uma correlação muito forte;

- Resultados entre $\mid 0,70$ e $0,89 \mid$ caracterizam uma correlação forte;

- Resultados entre $\mid 0,40$ e $0,699 \mid$ caracterizam uma correlação moderada;

- Resultados entre |0,20 e 0,399| caracterizam uma correlação fraca;

- Resultados entre |0 e 0,199| caracterizam uma correlação muito fraca.

\section{ANÁliSE E disCuSSÃo doS RESUltados}

Nesta seção são apresentados, analisados e discutidos, os resultados provenientes da relação entre os gastos públicos realizados pela UESB e o PIB dos municípios baianos relativos à sua inserção, bem como o comportamento destes gastos frente aos demais setores econômicos que integram a economia regional.

\subsection{Testes de Normalidade das Amostras}

Preliminarmente, apresentam-se os testes de normalidade das amostras, como pressupostos que envolvem a realização do teste de hipótese estatísticas. Nos testes foram verificadas as variáveis taxa de crescimento do PIB dos municípios e taxa de crescimento do gasto público da UESB. A normalidade dos dados foi apurada pelo teste de normalidade de Kolmogorov-Smirnov e Shapiro-Wilk (Tabela 1).

\section{Tabela 1- Testes de normalidade}

\begin{tabular}{l|llllll}
\hline \multirow{2}{*}{ Variáveis } & \multicolumn{6}{|c}{ Kolmogorov-Smirnova Shapiro-Wilk } \\
\cline { 2 - 7 } & Statistic & df & Sig. & Statistic df & Sig. \\
\hline TX_CRE_GPUB_UESB, 207 & 5 &, $200^{*}$ &, 968 & 5 &, 862 \\
\hline TX_CRE_PIB_MUNIC ,197 & 5 &, $200^{*}$ &, 962 & 5 &, 819 \\
\hline TX_CRE_VA_ADM &, 285 & 5 &, $200^{*}$ &, 889 & 5 &, 353 \\
\hline TX_CRE_VA_SERV &, 273 & 5 &, $200^{*}$ &, 923 & 5 &, 547 \\
\hline TX_CRE_VA_AGRO &, 172 & 5 &, $200^{*}$ &, 957 & 5 &, 790 \\
\hline TX_CRE_VA_IND &, 266 & 5 &, $200^{*}$ &, 803 & 5 &, 086 \\
\hline
\end{tabular}

Fonte: Elaborada pelos autores. a. Lilliefors Significance Correction 
Pode-se verificar por meio dos testes (Tabela 1) que os dados coletados apresentaram distribuição normal, uma vez que a hipótese nula de normalidade foi rejeitada ao nível de significância p> 0,05 para ambas as variáveis investigadas (gasto público da UESB; PIB dos municípios; valor adicionado do setor de serviços; valor adicionado do setor de agronegócio; valor adicionado do setor da indústria; e valor adicionado do setor da administração pública).

A normalidade dos dados também foi observada por meio do gráfico Q-Q Plot, a fim de se buscar uma melhor visualização da proximidade dos pontos derivados das variáveis em relação à reta de referência apresentada no gráfico (Figura 1).
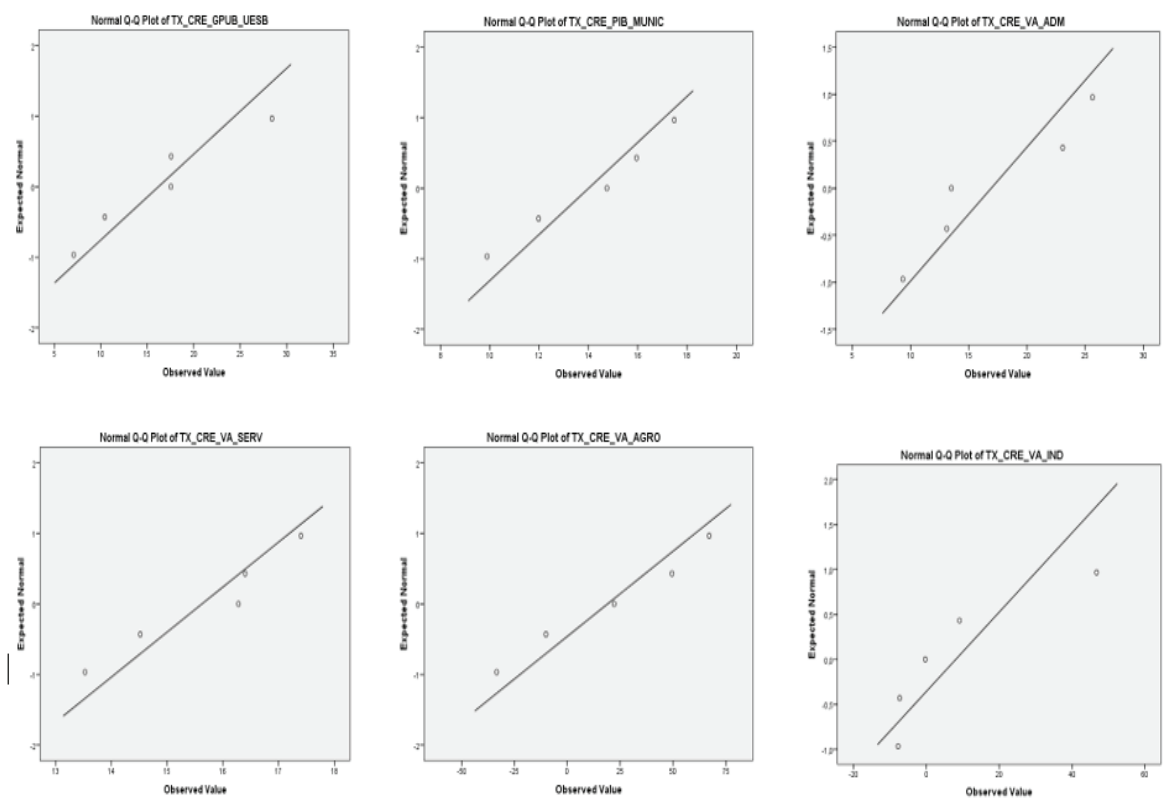

Figura 1 - Gráficos de normalidade Q-Q Plot

Fonte: Elaborada pelos autores.

Com base nos gráficos apresentados (Figura 1), os pontos que representam os valores das variáveis se aproximam da reta interseccional traçada entre o valor medido (no eixo horizontal) e a probabilidade acumulada (no eixo vertical). Apenas um dos pontos da variável taxa de crescimento do valor adicionado da indústria 
apresenta um curto afastamento da reta de distribuição, o que não gera comprometimento do requisito de significância.

Outro método de verificação da normalidade dos dados é por meio do escore padronizado (score Z) da assimetria (skewness) e da curtose (kurtosis), conforme debatido na literatura por Tabachnick e Fidell (1989). A Tabela 2 apresenta as estatísticas (Statistic), erro padrão (Std. Error) e escore Z ( $Z$ score) verificados na amostra.

Tabela 2 - Escore Z da assimetria e curtose

\begin{tabular}{|c|c|c|c|c|c|c|}
\hline \multirow[b]{2}{*}{ Variáveis } & \multicolumn{2}{|c|}{ Skewness } & \multirow[b]{2}{*}{ Z score } & \multicolumn{2}{|l|}{ Kurtosis } & \multirow[b]{2}{*}{ Z score } \\
\hline & Statistic & $\mid \begin{array}{l}\text { Std. } \\
\text { Error }\end{array}$ & & Statistic & \begin{tabular}{|l} 
Std. \\
Error
\end{tabular} & \\
\hline $\begin{array}{l}\text { TX_CRE_GPUB_ } \\
\text { UESB }\end{array}$ &,- 290 & 913 &,- 318 & ,548 & 2,000 & ,274 \\
\hline $\begin{array}{l}\text { TX_CRE_PIB_MU- } \\
\text { NIC }\end{array}$ &,- 431 & 913 &,- 472 & $-1,632$ & 2,000 &,- 816 \\
\hline TX_CRE_VA_ADM & 411 & 913 & 450 & $-2,434$ & 2,000 & $-1,217$ \\
\hline TX_CRE_VA_SERV &,- 558 & ,913 &,- 611 & $-1,580$ & 2,000 &,- 790 \\
\hline $\begin{array}{l}\text { TX_CRE_VA_ } \\
\text { AGRO }\end{array}$ &,- 375 & 913 &,- 411 & $-1,592$ & 2,000 &,- 796 \\
\hline TX_CRE_VA_IND & 1,694 & ,913 & 1,855 & 2,857 & 2,000 & 1,429 \\
\hline
\end{tabular}

Fonte: Elaborada pelos autores.

Considerando as estatísticas descritivas relacionados à assimetria (skewness) e curtose (kurtosis), apurados por meio do escore $\mathrm{Z}$ ( $Z$ score), a amostra apresentou uma distribuição normal, uma vez que os dados coletados indicaram um valor menor que 1,96 para um nível de significância p< 0,05 (TABACHNICK; FIDELL, 1989).

\subsection{Análise da Correlação Entre os Gastos Públicos da UESB, PIB dos Municípios e VA dos Setores Econômicos}

Apesar de ser constatada a normalidade dos dados, optou-se pelo uso do coeficiente de correlação de Spearman para análise das taxas anuais de crescimento do PIB dos municípios e de crescimento do gasto público da UESB, visto que o número de observações apresentaram $\mathrm{n}<30$ (PALLANT, 2011), mediante disponibilidade dos dados nas fontes pesquisadas. 
As séries de dados das taxas de crescimento, PIB dos municípios e gasto público da UESB, apresentados no Quadro 2, foram observadas em relação ao período de 2014 à 2018, sendo o ano de 2013 considerado apenas para referenciamento dos valores nominais e cálculo dos valores reais.

Quadro 2 - Comportamento das taxas de crescimento

\begin{tabular}{|l|l|l|}
\hline Ano & $\begin{array}{l}\text { Taxa de evolução do gasto } \\
\text { público da UESB (\%) }\end{array}$ & $\begin{array}{l}\text { Taxa de crescimento do VA da } \\
\text { Administração Pública (\%) }\end{array}$ \\
\hline $\mathbf{2 0 1 4}$ & 13,32 & 12,62 \\
\hline $\mathbf{2 0 1 5}$ & 5,09 & 7,68 \\
\hline $\mathbf{2 0 1 6}$ & 8,43 & 7,12 \\
\hline $\mathbf{2 0 1 7}$ & 8,42 & 8,39 \\
\hline $\mathbf{2 0 1 8}$ & 0,35 & 4,83 \\
\hline
\end{tabular}

Fonte: Elaborado pelos autores, a partir dos dados do IBGE (2020) e SEPLAN (2020).

Nesse sentido, preliminarmente, realizou-se a análise descritiva das variáveis por meio das medidas de centralidade e dispersão, além dos coeficientes de assimetria e curtose, conforme se demonstra na Tabela 3.

Com base na Tabela 3, observa-se que os desvios padrão das taxas de crescimento do gasto público da UESB (2,14243 \%) apresentam maior oscilação em comparação em relação à sua média $(7,122$ \%) do que a taxa de crescimento do PIB, desvio padrão (0,64191 \%) e média $(6,7680 \%)$. Em termos práticos, constata-se que a variávels de gasto público da UESB apresenta inconstância na representatividade da sua influência no crescimento econômico dos municípios, tendo por base o PIB.

Em seguida, realizou-se a análise da correlação entre as variáveis, considerando as taxas de crescimento dos gastos da UESB (X) e as taxas de crescimento do PIB dos municípios (Y) em teste de hipóteses associado à correlação bivariada. A matriz de correlação de Spearman entre as taxas de crescimento dos gastos da UESB e as taxas de crescimento do PIB dos municípios são demonstrados na Tabela 4. 
Tabela 3 - Análise descritiva preliminar

\begin{tabular}{|c|c|c|c|c|}
\hline \multicolumn{3}{|l|}{ Variáveis } & \multirow{2}{*}{$\begin{array}{l}\text { Statistic } \\
7,1220\end{array}$} & \multirow{2}{*}{$\begin{array}{l}\text { Std. } \\
\text { Error } \\
2,14243\end{array}$} \\
\hline \multirow[t]{13}{*}{ TX_CRE_GPUB_UESB } & \multicolumn{2}{|l|}{ Mean } & & \\
\hline & \multirow{2}{*}{$\begin{array}{l}95 \% \text { Con- } \\
\text { fidence } \\
\text { Interval } \\
\text { for Mean }\end{array}$} & $\begin{array}{l}\text { Lower } \\
\text { Bound }\end{array}$ & 1,1737 & \\
\hline & & $\begin{array}{l}\text { Upper } \\
\text { Bound }\end{array}$ & 13,0703 & \\
\hline & \multicolumn{2}{|c|}{ 5\% Trimmed Mean } & 7,1539 & \\
\hline & \multicolumn{2}{|c|}{ Median } & 8,4200 & \\
\hline & \multicolumn{2}{|l|}{ Variance } & 22,950 & \\
\hline & \multicolumn{2}{|c|}{ Std. Deviation } & 4,79061 & \\
\hline & \multicolumn{2}{|c|}{ Minimum } & ,35 & \\
\hline & \multicolumn{2}{|l|}{ Maximum } & 13,32 & \\
\hline & \multicolumn{2}{|l|}{ Range } & 12,97 & \\
\hline & \multicolumn{2}{|c|}{ Interquartile Range } & 8,16 & \\
\hline & \multicolumn{2}{|c|}{ Skewness } &,- 290 & ,913 \\
\hline & \multicolumn{2}{|l|}{ Kurtosis } & 548 & 2,000 \\
\hline \multirow{13}{*}{ TX_CRE_PIB_MUNIC } & Mean & & 6,7680 & ,64191 \\
\hline & \multirow{2}{*}{$\begin{array}{l}95 \% \text { Con- } \\
\text { fidence } \\
\text { Interval } \\
\text { for Mean }\end{array}$} & $\begin{array}{l}\text { Lower } \\
\text { Bound } \\
\end{array}$ & 4,9858 & \\
\hline & & $\begin{array}{l}\text { Upper } \\
\text { Bound }\end{array}$ & 8,5502 & \\
\hline & \multicolumn{2}{|c|}{ 5\% Trimmed Mean } & 6,7856 & \\
\hline & \multicolumn{2}{|l|}{ Median } & 7,1200 & \\
\hline & \multicolumn{2}{|l|}{ Variance } & 2,060 & \\
\hline & \multicolumn{2}{|c|}{ Std. Deviation } & 1,43536 & \\
\hline & \multicolumn{2}{|c|}{ Minimum } & 4,83 & \\
\hline & \multicolumn{2}{|l|}{ Maximum } & 8,39 & \\
\hline & \multicolumn{2}{|c|}{ Range } & 3,56 & \\
\hline & \multicolumn{2}{|c|}{ Interquartile Range } & 2,71 & \\
\hline & \multicolumn{2}{|c|}{ Skewness } &,- 431 & ,913 \\
\hline & \multicolumn{2}{|l|}{ Kurtosis } & $-1,362$ & 2,000 \\
\hline
\end{tabular}

Fonte: Elaborada pelos autores. 
Tabela 4 - Correlação de Spearman PIB municípios

\begin{tabular}{|c|c|c|c|}
\hline \multicolumn{2}{|l|}{ Spearman's rho } & TX_CRE_GPUB_UESB & TX_CRE_PIB_MUNIC \\
\hline \multirow{3}{*}{$\begin{array}{l}\text { TX_CRE_GPUB_ } \\
\text { UESB }\end{array}$} & $\begin{array}{l}\text { Correlation } \\
\text { Coefficient }\end{array}$ & 1,000 & 600 \\
\hline & Sig. (2-tailed) & & ,285 \\
\hline & $\mathrm{N}$ & 5 & 5 \\
\hline \multirow{3}{*}{$\begin{array}{l}\text { TX_CRE_PIB_MU- } \\
\text { NIC }\end{array}$} & $\begin{array}{l}\text { Correlation } \\
\text { Coefficient }\end{array}$ & 600 & 1,000 \\
\hline & Sig. (2-tailed) & ,285 & \\
\hline & $\mathrm{N}$ & 5 & 5 \\
\hline
\end{tabular}

Fonte: Elaborada pelos autores.

A partir da análise da matriz de correlação, percebe-se que não há uma correlação significativa entre o crescimento do gasto público da UESB e o crescimento do PIB dos municípios, no período de 2014 à 2018, já que o nível de significância indica um p>0,05 (0,285). Considerando a dimensão quantitativa das variáveis analisadas, salienta-se que as relações de causa e efeitos não foram observadas na pesquisa (FIGUEIREDO FILHO; SILVA JUNIOR, 2010).

Considerando a participação da UESB nos gastos públicos em relação aos gastos realizados pelas prefeituras dos municípios baianos de Vitória da Conquista, Jequié e Itapetinga, esperava-se constatar algum tipo de correlação signiticativa entre os gastos da UESB e o PIB dos municípios. Os gastos púbicos da UESB em relação aos gastos municipais foram registrados como superiores em $21 \%$ na análise da média simples ano a ano, no período de 2013 à 2020, conforme se evidencia na Figura 2. 


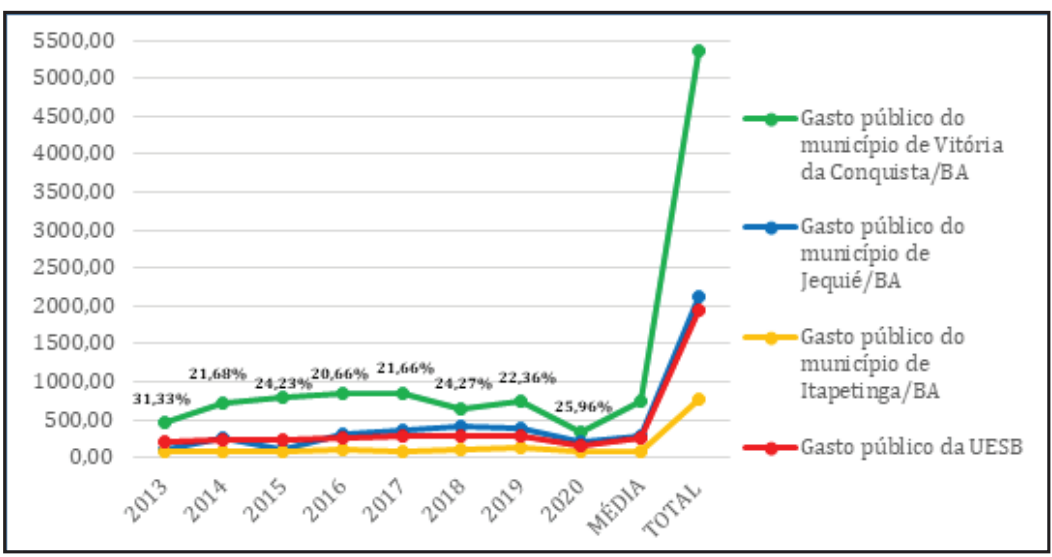

Figura 2 - Relação entre o gasto público da UESB e os gastos dos municípios, em bilhões.

Fonte: Elaborado pelo autor, a partir do SEPLAN (2020) e FIPLAN (2020).

No entanto, apesar da limitação estatística, buscou-se aprofundar as relações entre as taxas de crescimento do gasto público da UESB com os valores adicionados dos setores econômicos dos municípios, individualmente. Conforme observa-se na Figura 3, dada a participação dos gastos públicos da UESB no somatório dos valores adicionados do setor da administração pública e no somatório do VA total dos municípios, em relação ao período de 2013 a 2018, esperava-se evidenciar algum tipo de correlação.

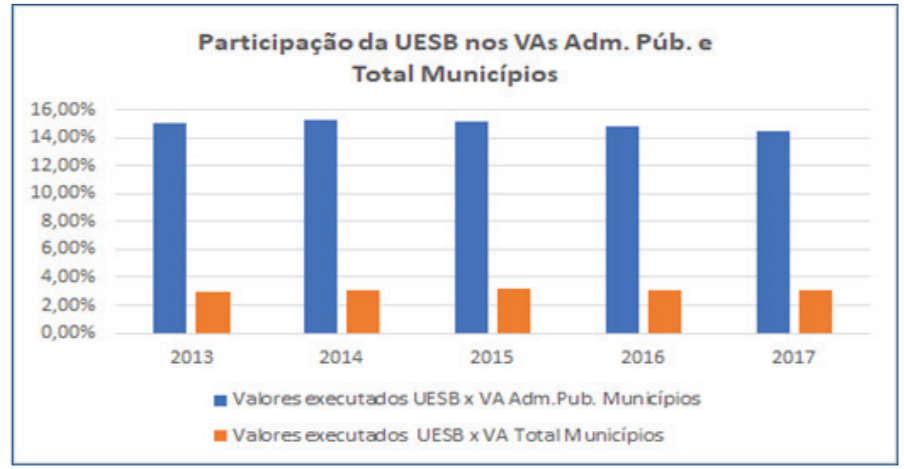

Figura 3 - Participação da UESB no VA da Administração Pública e VAs totais nos municípios, em bilhões.

Fonte: Elaborado pelo autor, a partir do SEPLAN (2020) e FIPLAN (2020). 
No entanto, as taxas de crescimento do gasto público da UESB não apresentaram correlação siginificativa com nenhuma variável relacionada aovalor adicionado dos setores econômicos dos municípios, conforme se demonstra na Tabela 5.

Tabela 5 - Correlação de Spearman VA setores econômicos

\begin{tabular}{|c|c|c|c|c|c|c|}
\hline \multicolumn{2}{|c|}{ Spearman's rho } & $\begin{array}{l}\text { TX_CRE_ } \\
\text { GPUB_UESB }\end{array}$ & $\begin{array}{l}\text { TX_CRE_ } \\
\text { VA_ADM }\end{array}$ & $\begin{array}{l}\text { TX_CRE_ } \\
\text { VA_SERV }\end{array}$ & $\begin{array}{l}\text { TX_CRE_ } \\
\text { VA_ } \\
\text { AGRO }\end{array}$ & $\begin{array}{l}\text { TX } \\
\text { CRE } \\
\text { VA_IND }\end{array}$ \\
\hline \multirow{3}{*}{$\begin{array}{l}\text { TX_CRE_ } \\
\text { GPUB_- } \\
\text { UESB }\end{array}$} & $\begin{array}{l}\text { Correlation } \\
\text { Coefficient } \\
\end{array}$ & 1,000 & 600 &,- 200 & -100 &, 500 \\
\hline & Sig. (2-tailed) & & ,285 & ,747 & ,873 & ,391 \\
\hline & $\mathrm{N}$ & 5 & 5 & 5 & 5 & 5 \\
\hline \multirow[t]{3}{*}{$\begin{array}{l}\text { TX_CRE_ } \\
\text { VA_ADM }\end{array}$} & $\begin{array}{l}\text { Correlation } \\
\text { Coefficient }\end{array}$ & 600 & 1,000 & 600 & ,500 &,- 100 \\
\hline & Sig. (2-tailed) & 285 & & 285 & ,391 & ,873 \\
\hline & $\mathrm{N}$ & 5 & 5 & 5 & 5 & 5 \\
\hline \multirow[t]{3}{*}{$\begin{array}{l}\text { TX_CRE_ } \\
\text { VA_SERV }\end{array}$} & $\begin{array}{l}\text { Correlation } \\
\text { Coefficient } \\
\end{array}$ &,- 200 & 600 & 1,000 & $900^{*}$ &,- 300 \\
\hline & Sig. (2-tailed) & ,747 & ,285 & & ,037 & ,624 \\
\hline & $\mathrm{N}$ & 5 & 5 & 5 & 5 & 5 \\
\hline \multirow[t]{3}{*}{$\begin{array}{l}\text { TX_CRE_ } \\
\text { VA_AGRO }\end{array}$} & $\begin{array}{l}\text { Correlation } \\
\text { Coefficient }\end{array}$ &,- 100 & ,500 & $900^{*}$ & 1,000 & 100 \\
\hline & Sig. (2-tailed) & 873 & ,391 & ,037 & &, 873 \\
\hline & $\mathrm{N}$ & 5 & 5 & 5 & 5 & 5 \\
\hline \multirow[t]{3}{*}{$\begin{array}{l}\text { TX_CRE_- } \\
\text { VA_IND }\end{array}$} & $\begin{array}{l}\text { Correlation } \\
\text { Coefficient }\end{array}$ &, 500 &,- 100 & -300 & 100 & 1,000 \\
\hline & Sig. (2-tailed) & ,391 &, 873 & ,624 & 873 & \\
\hline & $\mathrm{N}$ & 5 & 5 & 5 & 5 & 5 \\
\hline
\end{tabular}

A partir da análise da Tabela 5, nota-se que não há uma correlação significativa entre o crescimento do gasto público da UESB e 
os valores adicionados dos setores econômicos dos municípios, no período de 2014 à 2018, já que o nível de significância indica um p> 0,05 (VA setor administração pública 0,285; VA setor de serviços 0,747; VA setor de agropecuária 0,873; e VA setor industrial 0,391).

\section{Considerações finais}

A presente pesquisa teve por objetivo analisar a relação entre o comportamento dos gastos públicos da UESB e os indicadores econômicos dos municípios baianos de Vitória da Conquista, Jequié e Itapetinga (Produto Interno Bruto - PIB e Valores Adicionados VA's dos setores econômicos, tendo por base as teorias do gasto públicos e do desenvolvimento regional.

Para além da sua natureza no processo de formação e qualificação profissional, bem como na produção, valorização e disseminação do conhecimento e da inovação, que produzem efeitos na emancipação de pessoas, as universidades são influentes agentes do sistema econômico regional local. Na dinâmica da regionalidade, a universidade contribui para a circulação de recursos financeiros e estimula o crescimento econômico, contribuindo para a geração de emprego e renda.

No entanto, a partir das análises estatísticas, observou-se que apesar da relevância dos gastos públicos realizados pela UESB e sua possível influência na dinâmica econômica regional, estes não possuem correlação siginificativa e direta com o crescimento do PIB e com os VA's dos setores econômicos dos municípios baianos de Vitória da Conquista, Jequié e Itapetinga.

Apesar das evidências, é possível que a UESB exerça diversas influências sobre desenvolvimento econômico regional, em razão das vantagens provenientes das externalidades produzidas (spillovers), passíveis de verificação por meio dos efeitos multiplicadores dos gastos com pessoal, custeio e investimentos sobre os municípios, que provêm a comunidade regional com conhecimento, pesquisa e inovação.

Além disso, os efeitos dos gastos públicos da UESB, muito embora estatisticamente não estejam atrelados ao crescimento dos setores econômicos, certamente impactam os setores produtivos dada a sua força motriz na atração de novos negócios e ao fomento 
endógeno de novos empreendimentos ligados à indústria, comércio e serviços decorrentes das suas atividades e estímulos.

Como limitações desse trabalho salientam-se o uso apenas da análise do coeficiente de correlação dos dados levantados e a abordagem apenas no período de 2003 a 2018 para as taxas de crescimento de PIB e setores econômicos, visto serem esses os períodos com dados disponíveis. Para futuros estudos recomenda-se o aprofundamento da série de dados, inclusive de outras universidades baianas comparativamente, aplicando ferramentas estatísticas mais avançadas para análise dos dados.

\section{REFERÊNCIAS}

ALMEIDA, C. M. F. de. Universidade pública e seu entorno: bibliometria, estado das artes e evidências empíricas: lições para a universidade de Brasília no seu relacionamento com sua comunidade. 82 p. Dissertação (Mestrado profissional em Economia - Gestão Econômica de Finanças Públicas). Universidade de Brasília (UNB), Brasília, 2020.

BAHIA. Secretaria da Fazenda do Estado da Bahia. Portal da Transparência Bahia. Gastos diretos por unidade orçamentária. Disponível em: http://www.transparencia.ba.gov.br/ Pagamentos/. Acesso em: 25 ago. 2020.

BELLINGIERI, J. C. Teorias do desenvolvimento regional e local: uma revisão bibliográfica. RDE-Revista de Desenvolvimento Econômico, v. 2, n. 37, 2017.

BEZERRA, D. S. S., LIBONATI, J. J., OLIVEIRA MACEDO, M. R. G. de; BARROS RIBEIRO, M. V. J. de; BARROS RIBEIRO, M. T. J. de. Análise da relação entre receitas e despesas públicas e o desenvolvimento educacional dos municípios brasileiros baseada no índice FIRJAN. Enfoque: Reflexão Contábil, v. 37, n. 2, p. 89-106, 2018.

BONHO, F. T. A Universidade Federal do Pampa e o seu papel no desenvolvimento regional: estudo de caso do campus Caçapava do Sul/RS. 82 f. Dissertação (Desenvolvimento Regional), Faculdades Integradas de Taquara, Rio Grande do Sul, 2020.

BRAATZ, J.; GONÇALVES, R. da R.; FOCHEZATTO, A. Composição do gasto público estadual e crescimento municipal: Aplicação ao Rio Grande do Sul, 2004-2012. Revista Estudo \& Debate, v. 26, n. 3, 2019.

BRANDÃO, C. A. Dinâmicas e transformações territoriais recentes: o papel da PNDR e das políticas públicas não regionais com impacto territorial. IPEA, Texto para Discussão, 2019.

BRASIL. Ministério do Planejamento, Desenvolvimento e Gestão. Secretaria de Orçamento Federal. Manual Técnico de Orçamento - MTO. Brasília. 2020. Disponível em:< https://www1. siop.planejamento.gov.br/mto/doku.php/mto2020>. Acesso em: 20 ago. 2020.

BRASIL. Ministério da Transparência e Controladoria-Geral da União. Portal da Transparência. Gastos diretos por órgão executor. Disponível em: < http://www.portaltransparencia.gov.br/localidades>. Acesso em: 25 ago. 2020. 
BRESSER-PEREIRA, L. C. Desenvolvimento, sofisticação produtiva, valor-trabalho e salários. Nova Economia, v. 29, n. 1, p. 135-160, 2019.

CALIXTO, L. Estudos de caso sobre custos ambientais: ênfase nos procedimentos metodológicos. RAM - Revista de Administração Mackenzie, v. 10, n. 2, p. 87-109, 2009

CIRILLO, Marcelo Angelo; FERREIRA, Daniel Furtado. Extensão do teste para normalidade univariado baseado no coeficiente de correlação quantil-quantil para o caso multivariado. Revista de Matemática e Estatística, v. 21, n. 3, p. 67-84, 2003.

COSTA, G. P. C. L.; GARTNER, I. R. O efeito da função orçamentária alocativa na redução da desigualdade de renda no Brasil: uma análise dos gastos em educação e saúde no período de 1995 a 2012. Revista de Administração Pública, Rio de Janeiro, v. 51, n. 2. 2017.

FERREIRA, A; SANTOS, E. A. Expansão da universidade pública e o seu impacto na economia local: microevidências da ampliação dos campi da UFF em volta redonda. Revista Brasileira de Gestão e Desenvolvimento Regional, v. 14, n. 1, 2018.

FERREIRA, B.; RAPOSO, R. Evolução do (s) Conceito (s) de Desenvolvimento. Um Roteiro Crítico. Cadernos de Estudos Africanos, n. 34, p. 113-144, 2017.

FIPLAN. Sistema Integrado de Planejamento, Contabilidade e Finanças do Estado da Bahia. Secretaria da Fazenda do Estado da Bahia, 2020.

FITTIPALDI, I.; COSTA, S. F.; ARAÚJO, C. M. C. de. O gasto público federal brasileiro: um perfil incrementalista? 2017. Revista do Serviço Público, 68 (3), 611-630.

GIL, A. C. Como elaborar projetos de pesquisa. 4ª ed. São Paulo: Atlas, 2009

GUIMARÃES, Paulo Ricardo B. Análise de Correlação e medidas de associação. Universidade Federal do Paraná. Disponível em: $<$ https://docs. ufpr. br/ jomarc/correlacao. pdf $>$. Acesso em, v. 9, 2017.

HIROMOTO, M. H. Análise do efeito do gasto social dos governos federal, estadual e municipal sobre a pobreza no Brasil-1988 a 2010. Pesquisa e Planejamento Econômico - PPE. Rio de Janeiro, IPEA, v. 48, n. 1, p. 71-102, abr. 2018.

IBGE. Instituto Brasileiro de Geografia e Estatística. Índice de Preços ao Consumidor Amplo. Disponível em: < https:// https://sindusconpr.com.br/ipca-ibge-4023-p>. Acesso em: 19 dez 2020.

IBGE. Instituto Brasileiro de Geografia e Estatística. Deflator oficial IBGE. Disponível em: https://sidra.ibge.gov.br/tabela/6784. Acesso em: 19 dez 2020.

JESUS, J. A.; SPINOLA, N. D. Seis décadas da Teoria dos Polos De Crescimento: Revisitando Perroux. Revista de Desenvolvimento Econômico. V.17. N. 32. 2015.

LEOTTI, V. B.; COSTER, R.; RIBOLDI, J. Normalidade de variáveis: métodos de verificação e comparação de alguns testes não-paramétricos por simulação. Revista HCPA. Porto Alegre. Vol. 32, no. 2 (2012), p. 227-234, 2012.

LEOTTI, V. B.; BIRCK, A. R.; RIBOLDI, J. Comparação dos Testes de Aderência à Normalidade Kolmogorov-smirnov, Anderson-Darling, Cramer-Von Mises e Shapiro-Wilk por Simulação. Anais do 11ํㅗำ Simpósio de Estatística Aplicada à Experimentação Agronômica, 2005. 
LOPES, M. de M.; BRANCO, V. T. F. C.; SOARES, J. B. Utilização dos testes estatísticos de Kolmogorov-Smirnov e Shapiro-Wilk para verificação da normalidade para materiais de pavimentação. Transportes, v. 21, n. 1, p. 59-66, 2013.

MORGAN, D. L. Paradigms lost and pragmatism regained - methodological implications of combining qualitative and quantitative methods. Journal of Mixed Methods Research, Vol. 1, No. 1, 48-76, 2007.

MOTA, M. R. A.; GONÇALVES, S. da R. V. O papel da universidade pública frente ao Plano Nacional de Educação. TEXTURA-Revista de Educação e Letras, v. 22, n. 49, 2020.

NORMAN, Geoffrey R. et al. Biostatistics: the bare essentials. PMPH USA (BC Decker), 2008.

OLIVEIRA, J. A. J. Eficiência dos gastos públicos com ensino superior nas universidades federais brasileiras: uma aplicação da análise envoltória de dados. 126 p. Dissertação (Mestrado em Engenharia de Produção e Manufatura), Universidade Estadual de Campinas (UNICAMP), São Paulo, 2019.

ÖZTUNA, D.; ELHAN, A. H.; TÜCCAR, E. Investigation of four different normality tests in terms of type 1 error rate and power under different distributions. Turkish Journal of Medical Sciences, v. 36, n. 3, p. 171-176, 2006.

PANIZZI, W.; MEIRELLES, M.; HOPPE, L. As inter-relações entre universidade e o desenvolvimento regional e seus nexos com o conceito de rede. Revista Brasileira de Gestão e Desenvolvimento Regional, v. 14, n. 2, 2018.

RAMÍREZ-GUTIÉRREZ, Z.; BARRACHINA-PALANCA, M.; RIPOLL-FELIU, V. Eficiencia en la Educación Superior. Estudio Empírico em Universidades Públicas de Colombia y España. Revista de Administração Pública, v. 54, n. 3, p. 468-500, 2020.

REIS, A. G. B.; BUENO, N. P. O efeito dos gastos públicos sociais no crescimento econômico: uma análise comparativa entre os países membros da CEPAL e OCDE. Revista Gestão \& Tecnologia, v. 19, n. 1, p. 73-89, 2019.

ROCHA, R. H., MENEZES-FILHO, N., OLIVEIRA, A. P. D., KOMATSU, B. K. A relação entre o Ensino Superior público e privado e a renda e emprego nos municípios brasileiros. Pesquisa e Planejamento Econômico, v. 47, p. 39-69, 2017.

ROMANATTO, E., CRUVINEL, C. de E., ARAÚJO, E. S., SATEL, C. R. I. Caracterização econômica dos municípios goianos segundo valor adicionado dos setores de atividade. Revista Economia Ensaios, v. 30, n. 1, 2015.

SANTOS, E. L. Administração do desenvolvimento: um campo em busca da relevância ou a relevância em busca de um campo? 353 f. Tese (Doutorado em Administração). Universidade Federal da Bahia (UFBA), Salvador, 2014.

SANTOS, E. L.; SANTOS, R. S.; BRAGA, V. Administração do desenvolvimento: percepções e perspectivas da comunidade científica da AnPAD. Organizações \& Sociedade, v. 23, n. 77, p. 263-284, 2016.

SEPLAN. Secretaria do Planejamento do Estado da Bahia. Superintendência Estudos Econômicos e Sociais da Bahia. PIB Municipal. Disponível em: < https://www.sei.ba.gov.br/ index.php?option=com_content\&view=article\&id=561\&Itemid=335>. Acesso em 26 ago. 2020. 
TABACHNICK, B. G.; FIDELL, L. S. Using Multivariate Statistics. Harper Collins Publishers. New York, 1989.

TCM. Tribunal de Contas dos Municípios do Estado da Bahia. Portal do TCM/BA. Gastos por município. Disponível em: < https://www.tcm.ba.gov.br/consulta-de-despesas/>. Acesso em 26 ago. 2020.

YIN, Robert K. Estudo de Caso: Planejamento e métodos. Porto Alegre: Bookman editora, 2015.

Recebido em: 22-4-2021

Aprovado em: 2-12-2021

Avaliado pelo sistema double blind review.

Disponível em http://mjs.metodista.br/index.php/roc 